\title{
The Effect of Injectable Medication Procedure on the Level of Pain among Early Childhood patients
}

\author{
Farida A. K. Tahir \\ Department of Midwifery \\ Sulaimani Technical Institute \\ Sulaimani Polytechnic University \\ Sulaimani, Iraq \\ Farida.tahir@spu.edu.iq
}

\author{
Pary M. Azize \\ Nursing department \\ Sulaimani Technical Institute \\ Sulaimani Polytechnic University \\ Sulaimani, Iraq \\ Pary.azize@spu.edu.iq
}

\author{
Aram N. Abdul Razzaq \\ School of Nursing \\ University of Sulaimani \\ Sulaimani, Iraq \\ najataram@yahoo.com
}

\begin{abstract}
Volume 4 - Special Conference on Health \& Medical Sciences: Insight into Advanced Medical (ICHMS 2019)
\end{abstract}

DOI:

10.24017/science.2019 .ICHMS.3

Received:

14 June 2019

Accepted: 5 July 2019
Abstract

Children are often exposed to painful procedures during hospitalization. Validated age appropriate scales are available to assess pain among pediatric patients. The aim of this study is to find out the effect of injectable medication procedure on the level of pain among early childhood patients. This descriptive cross -sectional study was designed to assess the physiological and behavioral characteristic of pain using CRIES pain rating scale for both medical and surgical conditions, which contains (cry, spo2, heart rate, facial expression and sleep pattern). The study has been conducted for twelve weeks duration in the pediatric wards at medical and surgical hospitals in the Sulaimani city Kurdistan region of Iraq between $1^{\text {st }}$ March to $1^{\text {st }}$ Jun 2019. Fifty early childhood aged between (1 month- 3years) were participated and assessed pre injectable medication procedure and after. CRIES pain scales were applied to the children and shows that there is a highly significant differences between pain and the physiological, behavior characteristics between pre and after procedure in the pvalue < .00001 such as crying was developed from high pitch crying to inconsolable in post-procedure. Consumption of $\mathrm{O} 2$ also increased. In terms of heart rate was increased up to $20 \%$ than baseline, while, in the post-procedure, the change was more than $20 \%$ of the baseline. Facial expression was also changed to contracted face in the post procedure. Sleeping from short interval to absent sleeping 


\begin{abstract}
was shown. Significant variation between pre and postprocedure were assessed. Further variation between medical and surgical condition for mean score of every scale of CRIES were assessed. The implications of this study will impact on health professional's education; practice and future research are also recommending.
\end{abstract}

Keywords: pain, CRIES, pain rating tools, consequence of pain. Physiological sign, behavioral sign

\title{
1. INTRODUCTION
}

When exploring the history in the nature of pain, it is important to recognize that there are several definitions of pain, all acknowledging a multifaceted phenomenon of pain and over the past few decades grow to be the subject of many research studies [1].

Generally pain is measured using three methods: 1 . self-report, 2 . behavior, and 3 . physiological measurement, based on the age and cognitive development of the children [2]. During hospitalization, children are often exposed to painful procedures, several deferent scales have been validated to assess pain in specific populations of pediatric patients, Pain was assessed using indicator from CRIES pain score [3].

Pain in neonates has been often underreported and under management [4]. In addition, early exposure to pain can lead the way babies respond to pain later in life. Therefore, the health professionals should put into consideration the importance of assessing and managing pain clinically [5].

Pain Experience is a feeling that occurred as a consequence of diagnostic, such as a procedure pain or therapeutic interventions and also as a result of the disease process like infantile colic. Neonates cannot report their pain by word, which lead them to depend on others to assess and manage their pain. As a consequence, they might need a long term treatment. In order to provide an adequate management of pain, accurate assessment is essential. Some physiological and behavioral parameters are measured through CRIES pain rating tool, which aid a consistent pain management, so pain assessment is added to the list of vital sign and become the $5^{\text {th }}[6]$.

Several validate and reliable pain scales exists to measure acute pain in term and preterm neonates [7]. Most of these scales are designed in combination of both, behavioral indicators of pain, which include non verbal facial expression, some jerky body movements, and change in the pattern of crying. and/or physiological indicators of pain, which include ( tachycardia, change in respiratory rate, blood pressure and oxygen saturation [SaO2], vagal tone, palmer sweating, and plasma cortisol or catecholamine levels). CRIES is one of the behavioral scale that used to measure and observe these 5 parameters in letter such as $\mathrm{C}$ for crying, $\mathrm{R}$ for the requirement of oxygen supplementation (for $\mathrm{SaO} 2>95 \%$ ), I for increases in heart rate and or blood pressure, E for facial expression, and S for sleeplessness [8]. The aim of this study is to assess the effect of injectable medication procedure on the level and experience of pain among early childhood.

\section{Design of the study}

\section{METHODS AND MATERIALS}

Descriptive cross sectional study is implemented to assess the effect of injectable medication procedure on the level of pain among early childhood.

\section{Setting of the study}

The study was conducted in the pediatric medical and surgical wards in the Sulaimani city Kurdistan Region of Iraq

The study sample

50 early childhoods who admitted to pediatric hospitals in medical and surgical wards were monitored. The early childhood is with different categories (infants age of one month to 12 
months and toddlers' age from 1 year to 3 years) and condition of health is medical or surgical and both gender male and female.

\section{The study instrument}

CIRES pain scale were used for the purpose of the present study, the researcher assesses the effect of injectable medication procedure on the level of pain among early childhood pediatric hospital, it was based on extensive review of related literature and study. The data collection consists of two parts, which include demographic data, which consists of (age, gender and condition of the early childhoods) and CRIES pain scale which consists of (cry, spo2, heart rate, facial expression and sleep) find it in figure1

\section{The CRIES Neonatal Postoperative Pain Measurement Score}

\begin{tabular}{llll} 
& 0 & 1 & 2 \\
\hline Crying & No & High-pitched & Inconsolable \\
\hline Requires $\mathrm{O}_{2}$ for oxygen saturation $>95 \%$ & No & $<30 \%$ & $>30 \%$ \\
\hline Increased vital signs & $\mathrm{HR}$ and $\mathrm{BP}=$ or $<$ & $\mathrm{HR}$ or $\mathrm{BP} \uparrow<20 \%$ & $\mathrm{HR}$ or $\mathrm{BP} \uparrow>20 \%$ \\
& preoperative value & of preoperative value & of preoperative value \\
\hline Expression & None & Grimace & Grimace/grunt \\
\hline Sleeplessness & No & $\begin{array}{l}\text { The baby wakes at } \\
\text { frequent intervals }\end{array}$ & $\begin{array}{l}\text { The baby is } \\
\text { awake continuously }\end{array}$ \\
\hline
\end{tabular}

Figure 1: CRIES pain rating tool [8]

\section{Rating scales and scores}

CRIES pain scale are consisted of three-point scores $(0,1,2)$ using in every category This assessment tool is based on observations and objective measurements. It is monitored and gives score by a healthcare professional, such as a nurse or physician. Each parameter has two points to assign, with a rating of 0 for signs of no pain and a rating of 2 for signs of maximal pain. The items were rated and scored in figure 1.

The total score in CRIES pain scale if from 10 scores divided as the following:
$1-\quad 0=$ Relaxed and comfortable
2- 1 to $3=$ Mild discomfort
3- 4 to $6=$ Moderate pain
4- 7 to $10=$ Severe discomfort/pain

\section{Method of data collection}

Some demographic data were obtained such as ( age. gender and the child's health condition). Data. were applying on the patient level of pain in order to compeer the condition of child in terms of pain before and after injectable medication procedure collected through the use of CRIES pain scale.

\section{Statistical data analysis}

The obtained data of the respondents to the CRIES pain scale were entered to the computer and analyzed through the use of statistical package social science (SPSS version 22); the data analyzed was performed through the following approaches

\section{Descriptive statistics}

Such analysis was performed through the computation of the following

Frequencies distribution and percentage $\%=$ frequency / sample size $* 100$ 


\section{Mean of scores (MS)}

Mean of score was calculated from the ordinal data according to the three levels of scales as $(0,1,2)$ the highest of the scores indicated the severity of the pain. In the present study, the highest level of mean of scores indicates the high level of pain among the early childhoods in providing injectable medication procedure care. A mean score of $<0.66$ was considered mild pain, a mean score of 0.67 - 1.32 was considered moderate pain, and mean score of $1.33-2$ was considered severe pain.

M.S $=\frac{\sum \mathrm{fi} x \mathrm{i}}{\sum \mathrm{fi}}$

For mean to spo2 and heart rate per-procedure and post-procedure

Mean $=\frac{\text { sum }}{\mathrm{n}}$

The normal spo2 is less an equal 95

Mean of spo $2=\frac{95+96+97+98+99+100}{6}=97.5$

The average (Mean) of spo2 is equal to 97.5 .

This average or (mean) is the baseline for spo2 for calculate the per-procedure and postprocedure to assess CRIES pain scale.

Heart rate

Newborns (1-12) months (80-180) beat/minute

Toddler (1-3) years (80-140) beat/minute

The mean for heart rate (baseline) is

Mean of heart rate for infants ( 1 month to 12 months) equal to 130 beat/minutes.

Mean of heart rate for toddlers (1 year to 3 years) equal to 120 beat/minutes.

This average is the baseline for heart rate for calculate the per-procedure and post-procedure to assess CRIES pain scale

The formula to finding percent change is $\%$ change $=\frac{\text { pre-procedure-baseline }}{\text { baseline }}$ $\%$ change $=\frac{\text { post }- \text { procedure }- \text { basline }}{\text { baseline }}$

\section{RESULTS}

Table 1: Demographic data

\begin{tabular}{|c|c|c|c|c|}
\hline \multicolumn{2}{|l|}{ Demographic } & \multirow{2}{*}{$\begin{array}{l}\text { Frequency } \\
11 \\
\end{array}$} & \multirow{2}{*}{$\begin{array}{l}\% \\
22 \% \\
\end{array}$} & $\begin{array}{l}\text { Cumulative } \\
\text { Percent }\end{array}$ \\
\hline \multirow[t]{3}{*}{ Age } & 1-12 months & & & \multirow[b]{2}{*}{$78 \%$} \\
\hline & $1-3$ years & 39 & $78 \%$ & \\
\hline & Total & 50 & $100 \%$ & 100 \\
\hline \multirow[t]{3}{*}{ Gender } & Male & 27 & $54 \%$ & \multirow[t]{2}{*}{$54 \%$} \\
\hline & Female & 23 & $46 \%$ & \\
\hline & Total & 50 & $100 \%$ & $100 \%$ \\
\hline \multirow{3}{*}{$\begin{array}{l}\text { Condition } \\
\text { of patients }\end{array}$} & Medical & 26 & $52 \%$ & \multirow[t]{2}{*}{$52 \%$} \\
\hline & Surgical & 24 & $48 \%$ & \\
\hline & Total & 50 & $100 \%$ & $100 \%$ \\
\hline
\end{tabular}

Table 1 show that all the participants were early childhood patients aged between ( 1 month - 3 years., 1-12 months) are (11,22\%), and (39, 78\%) were children aged between 1-3 years. Regarding gender, $(27,54 \%)$ of them were male and $(23,46 \%)$ female. The conditions of children such as medical and surgical conditions were almost equal. 


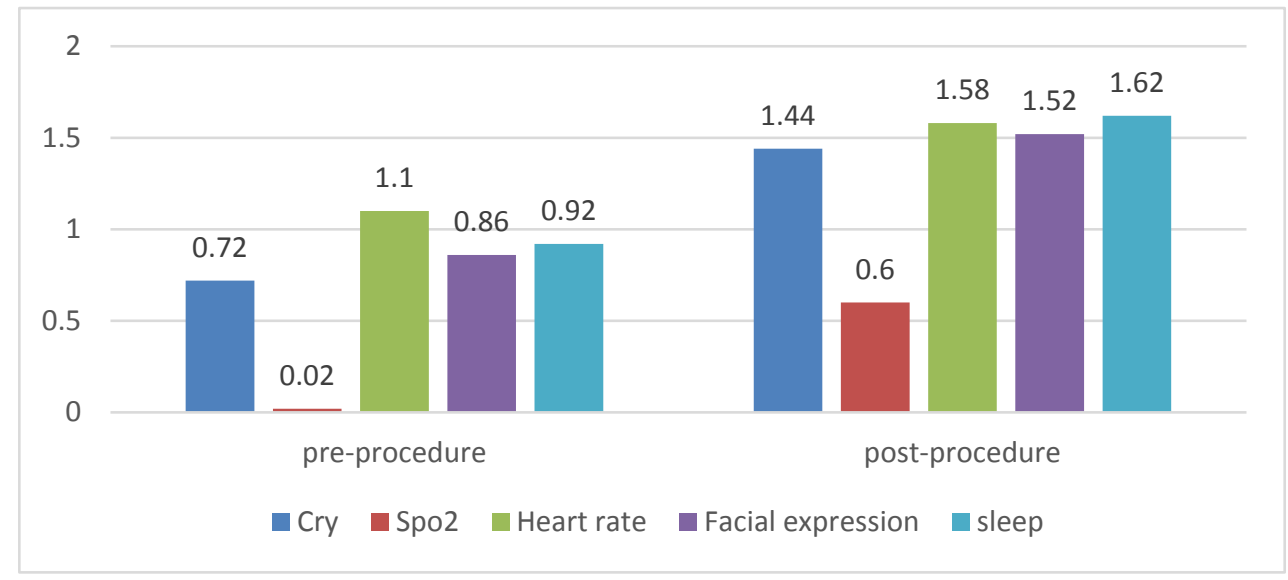

Figure 2: Mean consequence of pain

This bar graph shows mean of all categories of CRIES pain scale, which consist three score. A mean score of $<0.66$ was considered mild pain, a mean score of $0.67-1.32$ was considered moderate pain, and mean score of 1.33 - 2 was considered severe pain. The different between pre-procedure and post-procedure is significant changes. Pre-procedure for cry scale the mean show is on 0.72 that is on moderate pain, significant change on post-procedure were observed, the mean increased to 1.44, which mean severe pain. Spo2 on pre-procedure the mean of scale was 0.02 , which showed that the participant have relaxed with a little of pain. However, in the post-procedure the consumption of spo2 were raised to the mean score 0.6. In terms of heart rate scale, there was also a significant change in the mean scale in pre-procedure was 1.1 and post-procedure was 1.58 , which means the participant feels severe pain. Another scale is facial expression in pre-procedure the mean was 0.86 the participant feels moderate pain and post-procedure mean is 1.52 the participant feels severe pain.

The last scale is sleep pattern in pre-procedure the mean was 0.92 . While, in the postprocedure the mean was raised to 1.62, so participant feels severe pain.

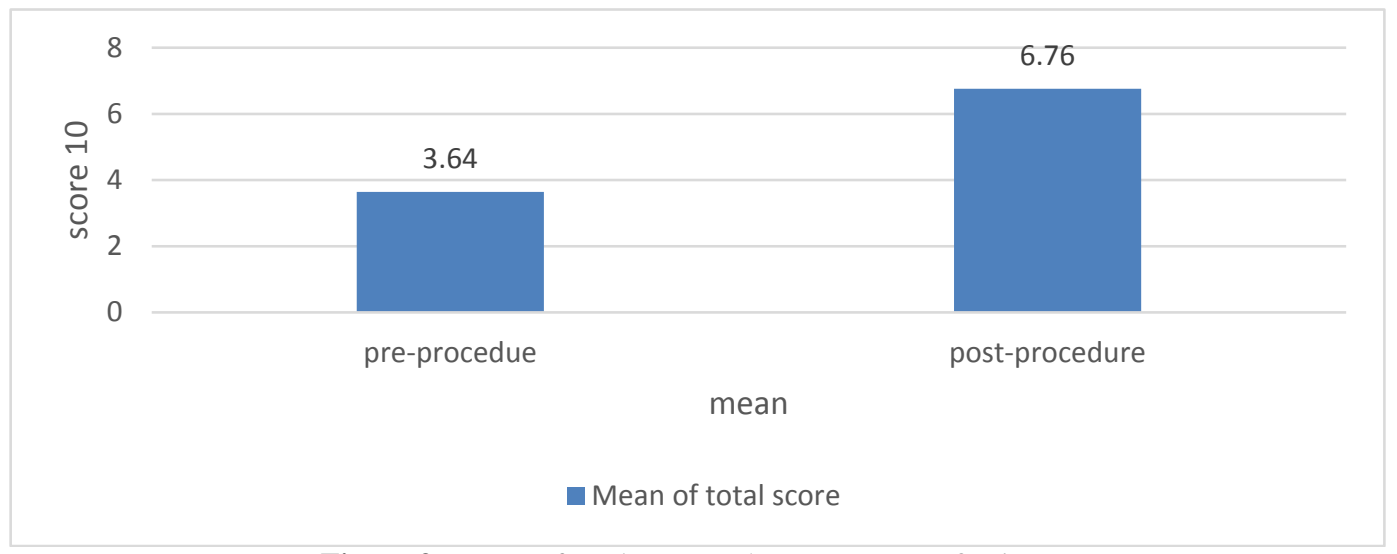

Figure 3: Mean of total score and consequence of pain

This bar graph shows the effect of Injectable medication procedure on the total score of the level of pain. The mean shows significant changes between pre-procedure and post-procedure. Pre-procedure mean score was 3.64 mean the participant feels mild pain. While, the total score of post-procedure were increased to 6.76 . 
Table 2: Differences between medical and surgical consequence of pain for total score

\begin{tabular}{|l|l|l|l|l|l|r|r|r|r|r|}
\hline \multirow{2}{*}{ Total score } & \multicolumn{2}{|c|}{$\begin{array}{c}\text { Relaxed } \\
0\end{array}$} & \multicolumn{2}{|c|}{$\begin{array}{c}\text { Mild } \\
1-3\end{array}$} & \multicolumn{2}{c|}{$\begin{array}{c}\text { Moderate } \\
4-6\end{array}$} & \multicolumn{2}{c|}{$\begin{array}{c}\text { Severe } \\
7-10\end{array}$} & \multicolumn{2}{c|}{ Total } \\
\cline { 2 - 12 } & F & \% & F & \% & F & \% & F & \% & F & Mean \\
\hline $\begin{array}{l}\text { Pre-procedure } \\
\text { Medical }\end{array}$ & 0 & 0 & 9 & 34.6 & 17 & 65.4 & 0 & 0 & 26 & 3.92 \\
\hline $\begin{array}{l}\text { Post-procedure } \\
\text { Medical }\end{array}$ & 0 & 0 & 2 & 7.7 & 10 & 38.5 & 14 & 53.8 & 26 & 6.8 \\
\hline $\begin{array}{l}\text { Pre-procedure } \\
\text { Surgical }\end{array}$ & 1 & 4.2 & 13 & 54.2 & 9 & 37.5 & 1 & 4.2 & 24 & 3.33 \\
\hline $\begin{array}{l}\text { Post-procedure } \\
\text { surgical }\end{array}$ & 0 & 0 & 0 & 0 & 9 & 37.5 & 15 & 62.5 & 24 & 6.7 \\
\hline
\end{tabular}

This table shows variation between medical condition and surgical condition of the total score of participant depending on CRIES pain scale. Medical condition, pre-procedure the patient feels moderate pain the percentage is $65.4 \%$ and post-procedure the participant feel severe pain the percentage is $53.8 \%$. Surgical condition, pre-procedure the participant feel mild pain the percentage is $54.2 \%$ and post-procedure the patients feel severe pain the percentage is $62.5 \%$. The table shows significant variation between medical and surgical condition

Table 3: Differences between medical and surgical consequence of pain for mean of scales

\begin{tabular}{|l|l|l|}
\hline \multicolumn{2}{|l|}{ Child's conditions } & Mean \\
\hline \multirow{2}{*}{ Medical } & Pre-procedure & 3.92 \\
\cline { 2 - 3 } & Post-procedure & 6.8 \\
\hline \multirow{2}{*}{ Surgical } & Pre-procedure & 3.33 \\
\cline { 2 - 3 } & Post-procedure & 6.7 \\
\hline
\end{tabular}

This table shows slight variations between medical condition and surgical condition because the mean score for every scales is changes between pre-procedure and post-procedure

Table 4: Differences between medical and surgical consequence of pain for total mean score

\begin{tabular}{|l|l|l|l|l|l|l|}
\hline Mean & Cry & Spo2 & HR & Facial & Sleep \\
\hline \multirow{3}{*}{ Medical } & Pre-procedure & 0.8 & 0.03 & 1.11 & 0.92 & 1 \\
\cline { 2 - 7 } & Post-procedure & 1.42 & 0.69 & 1.61 & 1.5 & 1.73 \\
\hline & Pre-procedure & 0.62 & 0 & 1.08 & 0.79 & 0.83 \\
\cline { 2 - 7 } & Post-procedure & 1.45 & 0.54 & 1.54 & 1.66 & 1.66 \\
\hline
\end{tabular}

This table shows slight variations between medical condition and surgical condition because the mean score for every scales is changes between pre-procedure and post-procedure This table shows a slight variation between medical and surgical condition. Significant changes between pre-procedure and post-procedure for each condition. Medical condition, pre-procedure mean score is 3.92 and the post-procedure mean score is 6.8 the score is significant increased. Surgical condition, pre-procedure mean score is 3.33 and the postprocedure mean score is 6.7 the score significant increased.

\section{DISCUSSION}

Children's pain is often under-reported, as a child's cognitive development is one of the factors that can affect a child's understanding of what is happening around them in relation to scoring their pain and using pain rating tools. Therefore, multidimensional behavioral pain 
scale are used in assessing the pediatric pain [9].

This study has been used CRIES pain scale, as it is an age appropriate scale that used to assess pain experience among the early childhood pre and post Injectable medication procedure. This is proved by some studies, which stated the validity and reliability of this instrument to use for pediatric populations especially early childhood [7, 10, and 11].

A significant variation of the level of pain between pre IMP and post has found in this study, as this proved by using CRIES to measure all the five parameters of the observational scale. Regarding the measurement of pain, crying is the first objective sign in CRIES tool, as this study has measured the pattern of crying before the IMP and after the procedure, found that the crying started from the beginning of the procedure with a high pitch crying to inconsolable crying until after of the procedure. Thus, one of the emotional responses to the stimuli among children is crying, as they could not be able to express pain by words [12]. The needle phobia among children and adolescence been studied clinically, they reported that the fear of injection is mostly associated with needle phobia during the needle puncture [13].

In terms of the saturation of oxygen and level of pain, SPO2 is the second parameter of CRIES that monitored in this study and found that Spo2 on pre-procedure the mean of scale was 0.02 , which showed that the participant have relaxed and a little pain been felt. However, in the post-procedure the consumption of spo2 were raised to the mean score of 0.6 . In the study of the applying CRIES scale in measuring the consequence of pain, mentioned that the level of oxygen are decreased during the painful procedure [3]. Regarding heart rate, this study found that heart rate scale is increased up to $20 \%$ than baseline and repeated in the postprocedure , which leads to increase to more than $20 \%$ than baseline. This is in consistent with the analyzed data during 2009 - 2010 from National Hospital Ambulatory Medical Care Survey in emergency department and found that pain can leads to tachycardia [14].

One of the non verbal indicators of pain experience among children is facial expression [15]. The present study depends on what Darwin's stated about the characteristic of pain through facial expression as follow:

[in pain] the mouth may be closely compressed, or more commonly, the lips are retracted, with the teeth clenched or ground together...the eyes stare wildly as if in horrified astonishment [16]

It consider as the most persistent in pre-procedure, as occasional grimace were presented, however, in the post-procedure that shape were changed to the contracted face.

Sleeping pattern was also monitored pre and post IMP, which was stated by the caregiver as a short interval and in the post-procedure was absent sleeping. Thus, stress and poor health are the two main interrelated factors that the children concerned, which affect the quality of sleep. As sleeping consider to be a significant indicator of poor health status [18]. To examine the association between pain and sleep, a large cross- sectional cohort study was conducted on cancer patients and conclude that, especially cancer pain may strongly affect sleeping and strongly concerned by the them and their caregivers [19].

A significant variation has shown between pre-procedure and post-procedure in every parameter of the CRIES total score

\section{CONCLUSION}

Pain is a fifth vital sign recently; therefore, well-tested multidimensional instruments may be preferable. The CRIES pain scale is beast scale for ages of early childhoods and the scale shows significant changes between pre-procedure and post-procedure this is mean the CRIES pain scale is fit for this age and accordant to the result found the effect of injectable medication procedure on the level of pain. Workshop for nurses and physician is required in order to educate them how to use the CRIES pain scale 


\section{REFERENCE}

[1]. K. Hanoch Kumar, P. Elavarasi. “ Definition of pain and classification of pain disorders”. Journal of Advanced Clinical \& Research Insights 3, 87-90. 2016.

[2]. Y Jarred, M Rebecca , M Sean, "Pain Outcomes: A Brief Review of Instruments and Techniques". Current Pain and Headache Reports. 13(1): 39-43. 2009.

[3]. H Ivan. , N Lawrence , G Donna , W Laura , H , "COVERS Neonatal Pain Scale: Development and Validation”. International Journal of Pediatrics 496719. 1-5. 2010.

[4]. B Kathryn , T Chambers. F Conrad. F Paula, L Margot. M Patrick et al., "Hospitalized children continue to report undertreated and preventable pain”. Pain Research \& Management. 19(4): 198-204, 2014.

[5]. W Nancy; P; Chris M.McCaffery, "Improving the Quality of Care Through Pain Assessment and Management”. Patient Safety and Quality: An Evidence-Based Handbook for Nurses, Agency for Healthcare Research and Quality (US); April.2008.

[6]. D María , O Begoña , S Alejandro, M A Juan , and F Inmaculada . “A review of chronic pain impact on patients, their social environment and the health care system” Journal of Pain Research. 9: 457-467, 2016.

[7]. $\mathrm{H}$ Linda.and E Elizabeth. "Measurement of Acute Pain in Infants:A Review of Behavioral and Physiological Variables”. Biological Research for Nursing. 17(1) 100-111. 2015.

[8]. S. W. Krechel and J. Bildner, "CRIES: a new neonatal postoperative pain measurement score. Initial testing of validity and reliability,” Paediatric Anaesthesia, vol. 5, no. 1, pp. 53-61, 1995.

[9]. S Rasha , R Savithiri , S Suzan "Pain in Children: Assessment and Nonpharmacological Management. International Journal of Pediatrics, 474838, 1- 112010.

[10]. B Alexandra i, M Kolia; P Dominique. "Pain Assessment in Newborns, Infants, and Children”. Pediatric Annals. 46(10): e387- e395. 2017.

[11]. M D Gleicia, PL Ana L de Aguiar, F Alline, M de Moura, M Vera Lúcia C Leitão . D MViviane. "Pain assessment scales in newborns: integrative review”. Revista Paulista de Pediatria. 32(4), 2014.

[12]. YK Chunawalla, MR Bohari, MN Bijle. "Correlation of Crying Pattern to Clinical Diagnosis of Children undergoing treatment”. International Journal of Contemporary Dentistry; 1:73-76. 2010

[13]. L. S Cook, “Needle phobia”. Journal of Infusion Nursing 39(5): 273-279. 2016.

[14]. DJ Elias . "Does Pain Lead to Tachycardia? Revisiting the Association Between Self-reported Pain and Heart Rate in a National Sample of Urgent Emergency Department Visits”, Mayo Clinic Proceedings. 90(8): 1165-1166. 2016

[15]. J J Chang , J Versloot, SR Fashler , KN McCrystal , KD Craig .”Pain assessment in children: validity of facial expression items in observational pain scales”. The Clinical Journal of Pain. 31(3):189-97. 2015.

[16]. C. Darwin The expression of emotion in man and animals. New York: Philosophical library; 1872/1955.

[17]. National sleep foundation. “ sleep \& disease, sleep \& lifestyles: sleep and pain” . 2019.

[18]. F H Patrick , G R Burel and ST Michael. "The association of sleep and pain: An update and a path forward”. The Journal of Pain. 14(12): 1539-1552. 2013.

[19]. EJ Stepanski, MS Walker, LS Schwartzberg, LJ Blakely, JC Ong, AC. Houts “The relation of trouble sleeping, depressed mood, pain, and fatigue in patients with cancer”. Journal of clinical sleep medicine:: official publication of the American Academy of Sleep Medicine. 5:132, 2009. 